\title{
«Sur mesure, les 7 unités du monde », exposition au musée des Arts et Métiers
}

Made to Measure: The World's 7 Units, an exhibition at the musée des Arts et Métiers

\section{Bruno Jacomy}

\section{OpenEdition} Journals

Édition électronique

URL : http://journals.openedition.org/artefact/3602

DOI : $10.4000 /$ artefact.3602

ISSN : 2606-9245

Éditeur :

Association Artefact. Techniques histoire et sciences humaines, Presses universitaires du Midi

\section{Édition imprimée}

Date de publication : 15 mars 2019

Pagination : 305-316

ISBN : 978-2-8107-0623-5

ISSN : 2273-0753

\section{Référence électronique}

Bruno Jacomy, " «Sur mesure, les 7 unités du monde », exposition au musée des Arts et Métiers », Artefact [En ligne], 9 | 2018, mis en ligne le 04 mars 2020, consulté le 27 novembre 2020. URL : http:// journals.openedition.org/artefact/3602 ; DOI : https://doi.org/10.4000/artefact.3602

\section{c) (i) $\Theta$}

Artefact, Techniques, histoire et sciences humaines est mise à disposition selon les termes de la Licence Creative Commons Attribution - Pas d'Utilisation Commerciale - Pas de Modification 4.0 International. 


\title{
«Sur mesure, les 7 unités du monde»
}

\author{
Exposition au musée des Arts et Métiers \\ Bruno Jacomy
}

\section{Résumé}

À l'occasion de la redéfinition, en novembre 2018, de quatre des sept unités de base du système international d'unités, le musée des Arts et Métiers présente une exposition sur le thème de la mesure. C'est l'opportunité, pour le musée, de sortir de ses réserves des instruments remarquables et peu connus du grand public, ainsi qu'un ensemble exceptionnel de mesures de capacité. Au long d'un parcours thématique, l'exposition déroule les différents aspects de la mesure, de celle de l'Homme à celle de la société, en mettant en avant les enjeux scientifiques, techniques, sociaux et économiques de la mesure, et ce depuis la mise en place du système métrique décimal sous la Révolution française.

\section{Mots-clés}

métrologie, poids et mesures, instruments de mesure, exposition, musée des Arts et Métiers, système international d'unités

99 Bruno Jacomy, « "Sur mesure, les 7 unités du monde", exposition au musée des Arts et Métiers », Artefact, 9, 2018, p. 305-316. 


\section{Made to Measure: The World's 7 Units, an exhibition at the musée des Arts et Métiers}

\section{Abstract}

On the occasion of the redefinition, in November 2018, of four of the seven base units of the international system of units, the musée des Arts et Métiers presents an exhibition on the subject of measurement. This is the opportunity for the museum to get out of its reserves some remarkable instruments and little known to the general public, as well as an exceptional set of capacity measures. Throughout a thematic journey, the exhibition presents the various aspects of the measurement, from that of Man to that of society, highlighting the scientific, technical, social and economic issues of measurement, and this since the introduction of the decimal metric system under the French Revolution.

\section{Keywords}

metrology, weights and measures, measuring instruments, exhibition, musée des Arts et Métiers, International System of Units 
A ujourd'hui, nous vivons dans une société où tout est mis en chiffres, quels que soient les domaines de l'activité humaine. Nous mesurons ou sommes mesurés à longueur de journée : la vitesse de notre voiture, le temps qui passe, la température, notre monnaie, les gigaoctets de nos ordinateurs... Ces actes sont tellement présents que nous n'y prêtons plus attention. Dès lors, la réalisation d'une exposition sur le thème de la Mesure s'est imposée comme une évidence ${ }^{1}$, d'autant que l'année 2018 voit l'abandon du dernier étalon matériel de référence pour la masse, le kilogramme " en platine iridié déposé au Pavillon de Breteuil à Sèvres ${ }^{2}$ ». Cette dématérialisation totale des unités est rendue indispensable pour permettre les développements futurs des technologies et des sciences. Ainsi, la $26^{\mathrm{e}}$ Conférence générale des poids et mesures, qui se tient en novembre 2018, considérant " qu'il est essentiel de disposer d'un Système international d'unités (SI) uniforme et accessible dans le monde entier, pour le commerce international, l'industrie de haute technologie, la santé humaine et la sécurité, la protection de l'environnement, les études sur l'évolution du climat, ainsi que la science fondamentale qui étaye tous ces domaines ${ }^{3}$ ", acte la redéfinition de quatre des sept unités de base du Système international : le kilogramme, le kelvin, la mole et l'ampère. Le mètre, la seconde et la candela garderont leurs définitions actuelles.

Le Conservatoire des arts et métiers et le système métrique décimal sont tous deux des créations de l'époque révolutionnaire ${ }^{4}$. L'esprit des Lumières qui soufflait alors a inspiré l'unification des mesures comme un élément indispensable à la diffusion des connaissances et aux échanges, qu'ils soient scientifiques, commerciaux ou techniques. Les savants de la Révolution, à l'origine de ces deux initiatives, ont agi en visionnaires ; ils étaient convaincus que la mesure serait l'un des grands enjeux de la société

\footnotetext{
1. L'exposition Sur mesure, les 7 unités du monde, est présentée au musée des Arts et Métiers (60 rue Réaumur, Paris $3^{\mathrm{e}}$ ), du 16 octobre 2018 au 5 mai 2019. Un catalogue est édité à cette occasion : Sur mesure, les 7 unités du monde, Paris, musée des Arts et Métiers/RMN-GP, 2018 (bilingue français/anglais). Articles de Bruno Jacomy, Maguelonne Chambon et Marc É. Himbert, Bernadette Bensaude-Vincent, Jean-Marc Lévy-Leblond, Céline Fellag Ariouet et Richard S. Davis.

2. Ce prototype a succédé en 1889 au décimètre cube d'eau pure (Comptes rendus, $1^{\text {re }}$ CGPM, 1889, complété par Comptes rendus, $3^{e}$ CGPM, 1901), et il n'aura plus cours à l'issue de la $26^{e}$ Conférence générale des poids et mesures.

3. Convocation de la Conférence générale des poids et mesures (26 réunion), Versailles 13-16 novembre 2018, Sèvres, Bureau international des poids et mesures, février 2018.

4. Ken Alder, Mesurer le monde : 1792-1799, l'incroyable histoire de l'invention du mètre, Paris, Flammarion, 2005.
} 
industrielle qui pointait alors. Bien sûr, ils ne pouvaient imaginer que, plus de deux cents ans plus tard, cette question garderait une place aussi cruciale dans la société mondiale.

De fait, la mesure est fondatrice de nos sociétés 5 . Dès que des hommes se sont rassemblés en communautés, les questions du partage des biens, du comptage des animaux, du calcul de la surface des terres se sont posées. Toute la vie sociale a pu s'organiser, avec plus ou moins d'harmonie, grâce à l'adoption d'unités de mesure communes, d'outils d'évaluation ou de comptage adaptés, de méthodes de calcul partagées.

\section{Un voyage dans le monde de la mesure}

En prenant la décision de créer cet événement à destination du grand public, à l'initiative du Laboratoire national de métrologie et d'essais (LNE), le musée des Arts et Métiers réaffirme sa volonté de s'inscrire dans le monde contemporain, de montrer qu'un musée n'est pas simplement tourné vers le passé, mais que sa mission est avant tout de s'intéresser aux questions qui agitent la société de son temps. C'est pourquoi cette exposition n'est pas à proprement parler une exposition historique. Bien sûr, elle s'appuie sur les prestigieuses collections du musée, mais elle s'attache avant tout à prendre en considération les préoccupations des citoyens du $\mathrm{XXI}^{\mathrm{e}}$ siècle et les réalités d'un monde qui tend à quantifier jusqu'à nos activités les plus personnelles.

L'exposition Sur mesure, les 7 unités du monde propose donc un voyage au pays de la mesure, en partant de l'Homme et de son corps, à l'origine des étalons de longueur usuels, pour se terminer avec la société, et notamment les questions que pose l'irruption des données quantifiées à tous les niveaux. Dans ce parcours thématique, nous avons choisi d'inviter les visiteurs à pénétrer au cœur de la métrologie, dans les dimensions les plus variées de cette science de la mesure : technique et scientifique bien sûr, mais aussi artistique, symbolique ou littéraire.

Les visiteurs sont accueillis, dès leur entrée dans l'exposition, par une évocation de la présence constante de la mesure dans la vie quotidienne :

5. Jean Perdijon, La mesure : histoire, science et philosophie, Paris, Dunod, 2004 ; Franck JedrZejewski, Histoire universelle de la mesure, Paris, Ellipses, 2002. 
notre consommation de gaz, notre poids, le temps qui passe, la température, notre monnaie... Mais ces mesures pratiques n'ont d'intérêt que si nous partageons les mêmes unités, les mêmes modes de mesurage. C'est la raison première qui a présidé à la mise en place, il y a maintenant plus de deux siècles, d'un système cohérent d'unités et d'étalons, toujours en usage mais sans cesse précisé, complété, disséminé.

Le premier objet historique qui ouvre l'exposition est aussi curieux que représentatif du rôle joué par le Conservatoire des arts et métiers dans l'aventure du système métrique. Il s'agit du creuset $^{6}$ qui a servi, le 13 mai 1874 , à couler une masse de près de $300 \mathrm{~kg}$ d'un alliage contenant $90 \%$ de platine et $10 \%$ d'iridium, l' "alliage du Conservatoire ${ }^{7}$ ", pour réaliser les premiers prototypes internationaux du mètre à profil en X. L'opération, dirigée par Henri Tresca (1814-1885), sous-directeur du Conservatoire, son fils Gustave (1846-1918), Henri Sainte-Claire Deville (1818-1881) et Henri Debray (1827-1888), s'est déroulée à deux pas de la salle où se tient l'exposition, dans la cour Vaucanson, devant une délégation de représentants de Russie, d'Angleterre, de Belgique et des Pays-Bas (Fig. 1).

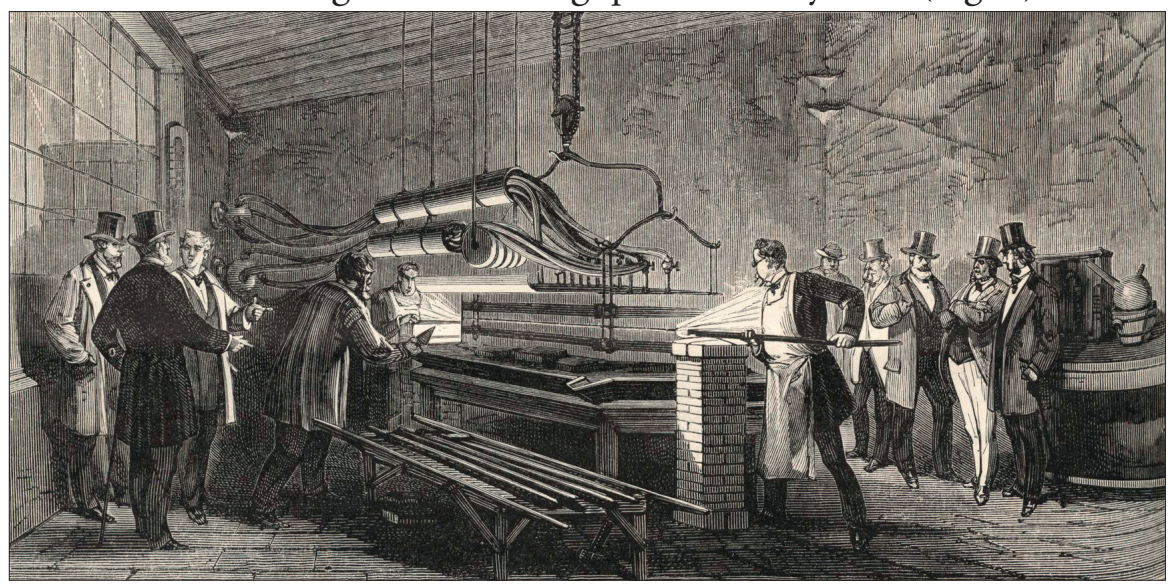

Fig. 1 - « La coulée de l’alliage de platine et d'iridium nécessaire pour forger les prototypes internationaux du mètre au Conservatoire des arts et métiers, 13 mai 1874 »

Le Monde illustré, 6 mars 1875, p. 164. Dessin de M. Miranda, gravé par Louis Joseph Amédée Daudenarde.

6. Creuset en calcaire à gros grain de Saint-Waast (Nord), vers 1880. Reproduction du creuset original de 1874, détruit pour l'extraction de l'alliage (musée des Arts et Métiers-Cnam, inv. 9478). 7. Louis André, "L'alliage du Conservatoire ", La Revue, n 5, musée des Arts et Métiers-Cnam, 1993, p. 39-42. 


\section{La mesure de l'Homme}

La première partie de l'exposition confronte le visiteur à la question de sa propre mesure. La connaissance de notre taille, de notre poids, de notre température ou de notre tension artérielle est en effet un élément essentiel à la maîtrise de notre santé. C'est l'occasion de rappeler que les unités de longueur, et donc de capacité, ont été, de l'Antiquité jusqu’à la Révolution française, établies à partir de nos mensurations. Mais, si la mesure de ces grandeurs corporelles est objective, l'utilisation qui en est faite peut être abusive, voire discriminatoire. La présentation du goniomètre de Paul Broca (1824-1880) nous rappelle ainsi que la mesure de la forme du crâne a pu servir, au XIX ${ }^{e}$ siècle, à classer les êtres humains selon une échelle d'intelligence ${ }^{8}$. Ce sont souvent par de menus détails que les objets présentés dans les musées révèlent leur dimension humaine ou sociale.

Ainsi, les visiteurs découvrent aussi un dynamomètre à la fois banal et très particulier. Cet instrument destiné à mesurer la force musculaire de la main, encore proche de celui inventé vers 1790 par Edme Regnier (1751-

3101825 ) pour mesurer la force des chevaux, existe sous diverses formes dans les réserves du musée des Arts et Métiers. Celui qui est exposé, emprunté à la Maison du patrimoine et de la mesure ${ }^{9}$, nous plonge dans notre histoire récente. Il a en effet été utilisé pour mesurer les capacités musculaires des hommes en vue du recrutement de mineurs dans les villages d'Afrique du Nord à la sortie de la Seconde Guerre mondiale. En fonction de leur aptitude, ces hommes pouvaient être estimés aptes à venir travailler dans les mines du bassin houiller de Saint-Étienne. De nombreux objets présentés ou conservés dans les réserves des musées sont ainsi nantis d'une histoire individuelle, voire intime, que les conditions d'entrée dans les collections ne permettent hélas pas toujours de mettre en évidence (Fig. XIX, cahier couleur).

\footnotetext{
8. Ce modèle « de voyage » du goniomètre facial et latéral, inventé par Paul Broca et datant de 1864, a été prêté par l'Institut de paléontologie humaine, Muséum national d'histoire naturelle, Paris. Voir Paul Broca, Instructions générales pour les recherches anthropologiques à faire sur le vivant, $2^{\mathrm{e}}$ éd., Paris, G. Masson, Société d'anthropologie de Paris, 1879, p. 39-43.

9. La Maison du patrimoine et de la mesure (rue du Stade, 42350 La Talaudière) présente une très riche collection de poids et mesures, essentiellement du département de la Loire, ainsi que des ensembles très complets d'instruments de vérification et de contrôle. Voir Trois siècles de l'histoire de la mesure, La Talaudière, Maison du patrimoine et de la mesure, 2001.
} 


\section{L'acte du mesurage}

Mesurer, c'est par définition comparer une grandeur physique inconnue avec une grandeur de même nature prise comme référence, à l'aide d'un instrument ${ }^{10}$. Il faut pour cela partager les mêmes unités et les mêmes méthodes de mesurage. Lopération est simple quand on dispose d'un instrument qui donne directement la valeur numérique, comme le mètre à ruban, mais ce n'est pas possible dans la plupart des cas. Les grandeurs sont souvent évaluées par une opération indirecte, sans que nous en soyons toujours conscients. Quand nous lisons la température sur un thermomètre à alcool, nous mesurons en fait la longueur d'une colonne de liquide que la dilatation fait varier. De même, dès l'Antiquité, la mesure de la surface des champs s'est faite grâce à des instruments de mesure d'angle associés à des méthodes de triangulation. Une petite expérience de mesure de la hauteur d'une tour à l'aide d'un quadrant est ainsi proposée aux visiteurs de l'exposition.

On voit également combien il est difficile de s'accorder sur les valeurs de certains phénomènes, telles les couleurs en peinture ou en photographie, même en recourant à des palettes normalisées. L'exposition offre ainsi l'occasion de sortir des réserves des objets méconnus, tel le colorimètre à double lunette inventé et construit par Charles-Félix CollardeauDuheaume (1796-1869) vers $1870^{11}$. Cet appareil, fondé sur un système de comparaison optique de la teinte à mesurer par rapport à une teinte de référence, a notamment été utilisé au XIX ${ }^{\mathrm{e}}$ siècle pour détecter la fraude dans le commerce des substances tinctoriales, comme le rocou.

\section{Unités et étalons}

Au cœur de l'exposition sont évoquées les unités de base et les unités dérivées du Système international. Cette partie est introduite par la présentation

\footnotetext{
10. Laurence Moulinier, Line Teisseyre-Sallmann, Catherine Verna et Nicolas Weill-Parot (dir.), La juste mesure : quantifier, évaluer, mesurer, entre Orient et Occident (VIII'-XVII' siècle), SaintDenis, Presses universitaires de Vincennes, 2005. Jean-Claude Beaune (dir.), La Mesure : instruments et philosophies, Seyssel, Champ Vallon, 1994.

11. Musée des Arts et Métiers-Cnam, inv. 8757. Voir la "Description d'un colorimètre à double lunette, par M. Collardeau [...] ", Bulletin de la Société d'encouragement pour l'industrie nationale, $37^{\mathrm{e}}$ année, février 1838, p. 54-58, pl. 720.
} 
spectaculaire d'un ensemble d'une centaine de mesures de capacités, choisies parmi les nombreux étalons rassemblés au cours $\mathrm{du} \mathrm{XIX}^{\mathrm{e}}$ siècle par le Conservatoire en échange de la diffusion d'étalons français. Les pièces exposées proviennent d'Allemagne, d'Amérique, de Tunisie ou de Russie, en tout d'une douzaine de pays différents, et ont été conçues pour les matières les plus variées : liquides, vin, grains et autres matières sèches... Elles sont aussi constituées des matériaux les plus divers : fer, bois, laiton, tôle étamée... Cette extraordinaire variété montre bien l'impérieuse nécessité de se donner des unités communes si l'on veut faciliter les échanges, notamment entre nations.

Cette partie centrale de l'exposition, fidèle à la tradition de transmission du savoir du Conservatoire, vise à expliquer en quoi consiste le Système international d'unités, ce que ces dernières représentent, comment elles ont été définies au cours de notre histoire et les enjeux scientifiques de leur évolution. En effet, au cours de cette révision fondamentale, les quatre unités qui font l'objet de définitions nouvelles - le kilogramme, l'ampère, le kelvin et la mole -, seront déterminées par rapport à des constantes physiques universelles telles que les constantes de Planck, de Boltzmann, d'Avogadro ou la charge élémentaire ; elles seront ainsi mieux adaptées à l'évolution des techniques et des sciences. Pour chacune des sept unités de base, des interviews filmées de scientifiques du Laboratoire national de métrologie et d'essais ou du Laboratoire commun de métrologie (LNECnam) facilitent la compréhension des nouvelles définitions d'unités, avec la disparition des étalons matériels, l'évolution des méthodes de mesure, etc. (Fig. XX, cahier couleur).

Le chapitre sur les unités, complété d'un audiovisuel retraçant l'histoire de la mise en place du système international, se termine avec un " cabinet des curiosités métrologiques ", où l'on peut découvrir des réminiscences d'unités anciennes ou de bien étranges instruments montrant l'étendue du champ de la mesure, comme un stéréoesthésimètre pour mesurer l'acuité tactile $^{12}$ ou un conformateur de chapelier pour prendre la forme et la mesure de la tête ${ }^{13}$ (Fig. XXI, cahier couleur).

12. Appareil conçu par Édouard Toulouse et Nicolas Vaschide, construit par G. Boulitte vers 1905. Prêt du Musée d'histoire naturelle, Lille, inv. 997-4.27. Voir La mesure de l'homme, instruments et tests du Musée d'histoire naturelle de Lille. Lille, Musée d'histoire naturelle, Paris, Somogy, 2002.

13. Instrument fabriqué par la maison Allié-Maillard, Paris, vers 1930. Prêt de l'Atelier-musée du chapeau, Chazelles-sur-Lyon. 


\section{De l'étalon à l'étal}

Si les unités, surtout par leurs nouvelles définitions, permettent des mesures exactes et reproductibles, elles ne constituent pas des étalons pratiques pour les usages courants. Or, les instruments employés dans les laboratoires ou chez les commerçants doivent être conformes à la réglementation afin que les échanges soient justes et que les fraudes ou erreurs soient limitées. Il est donc nécessaire de disposer d'une chaîne de traçabilité fiable pour assurer la certification des instruments utilisés.

Le Laboratoire national de métrologie et d'essais est notamment en charge de vérifier la validité de cette chaîne ininterrompue d'étalons et de procédures qui relie les étalons officiels aux instruments posés sur l'étal du marchand, aux laboratoires de recherche ou aux ateliers industriels de production. Bien avant même que ne soit établi le système métrique, des vérificateurs avaient la charge de contrôler, chez les commerçants, que leurs mesures de masse et de volume étaient bien conformes aux étalons royaux. Deux objets remarquables rappellent dans l'exposition ce rôle essentiel du contrôle : une armoire grillagée comportant vingt étalons de mesures à huile de la ville de Paris de $1741^{14}$ et une mallette de vérificateur du milieu du $\mathrm{XIX}^{\mathrm{e}}$ siècle $^{15}$. Tous deux témoignent de ce contact direct avec le commerçant final. Les sangles et le rembourrage de la première attestent l'usage itinérant de l'objet, lorsque le vérificateur allait à pied jusqu'à l'échoppe du maître chandelier huilier ; le soin et la fonctionnalité du second témoignent de la parfaite adaptation de la mallette à la charge d'un vérificateur parcourant à cheval la campagne drômoise vers 1855 .

\section{Usages et représentations de la mesure}

À partir d'un phénomène, d'une réalité, d'un objet, la mesure produit des valeurs numériques. Mais souvent, il est bien difficile de se représenter ces grandeurs, soit parce que les unités ne nous sont pas familières, soit parce

\footnotetext{
14. Ensemble datant de 1741 provenant de la galerie des Poids et Mesures du ministère de l'Agriculture et du Commerce, entré dans les collections du Conservatoire des arts et métiers en 1848 (musée des Arts et Métiers-Cnam, inv. 3254).

15. Nécessaire de vérificateur à cheval, fabriqué par Parent, à Paris, vers 1855. Une longue bride en cuir permet de le suspendre aux fontes de la selle. Prêt de la Maison du patrimoine et de la mesure, inv. 1104.
} 
qu'elles se situent dans des valeurs extrêmes. Par ailleurs, il est souvent nécessaire de garder la trace d'une mesure ponctuelle, qu'il s'agisse d'une expérience sur les particules élémentaires ou d'un phénomène qui varie, comme dans le cas de la météorologie.

C'est pour rendre compte de cette innovation importante, dans le monde de la métrologie, qu'est présenté dans l'exposition l'anémomètre créé en 1753 par Louis Léon Pajot (1678-1754), comte d'Ons-en-Bray ${ }^{16}$, le plus ancien instrument scientifique connu comportant un système d'enregistrement des données. Il côtoie une autre pièce remarquable, le prédicteur de marée de lord Kelvin (1824-1907) ${ }^{17}$. Grâce à un jeu de câbles et de poulies, le Tide Predictor assure la somme des huit premières composantes de la courbe d'évolution des marées. Celui du musée des Arts et Métiers qui a été utilisé par le Service hydrographique et océanographique de la Marine est l'un des plus anciens qui subsistent (Fig. XXII, cahier couleur).

La mesure est aussi fréquemment traduite en graphes, courbes, images numériques. Cette mise en forme des données suppose dans de nombreux 314 cas une interprétation, qui doit être la plus juste possible. Ceci ne nous met pas à l'abri d'erreurs, voire de tromperies, lorsque des données sont oubliées ou dissimulées, ou quand des échelles sont volontairement tronquées. La mesure est bien une réalité objective, mais il nous faut savoir en déjouer certains pièges.

\section{Mesure et société}

Les usages de la mesure dans la vie sociale sont multiples, dans l'industrie et la recherche scientifique bien sûr, mais de plus en plus dans les mesures d'opinion ou de comportement, avec les statistiques et le traitement des

16. Anémomètre enregistreur marquant la direction et la vitesse du vent, mis au point par Louis Léon Pajot, comte d'Ons-en-Bray et construit en 1753 (musée des Arts et Métiers-Cnam, inv. 5608). Voir Bruno Jасомy, "L'anémomètre de Pajot d'Ons-en-Bray ", La Revue, n 30, musée des Arts et Métiers, 2000, p. 30-46.

17. Machine à prédire la marée, dite "Tide Predictor n ${ }^{\circ} 3$ ", conçue en 1872 par William Thomson, lord Kelvin, et construite par James White en 1881 (musée des Arts et Métiers-Cnam, inv. 21936). Son fonctionnement est détaillé dans : Pierre Devaux, "Les Machines à prédire les marées ", La Nature, 1936, $2^{\mathrm{e}}$ semestre, p. 449-457. 
données en masse ${ }^{18}$. La dernière partie de l'exposition conduit justement les visiteurs vers les questions posées, dans notre société actuelle, par la diffusion de données individuelles ou les réseaux sociaux. L'exposition s'est ouverte sur la mesure de l'Homme, avec les questions de santé individuelle; elle se clôt avec la santé partagée, illustrée par des objets connectés - capteur de glycémie, balance ou fourchette électronique - dont les résultats de mesure peuvent parfois dépasser notre propre usage et être exploitées à notre insu.

Vivre en société suppose une gestion de tout ce qui concerne les citoyens : aménager l'espace, construire des habitations, des hôpitaux et des écoles, élaborer des politiques familiales, programmer des formations... Pour anticiper les évolutions de la société, il est indispensable de connaître au plus près la composition de la population, d'en mesurer toutes les composantes. C'est pour cette raison que le dernier objet exposé est l'extraordinaire machine à statistiques qu'Herman Hollerith (1860-1929) a mise au point en 1889 pour permettre au Census Bureau américain de connaître l'état de la population des États-Unis ${ }^{19}$. Il fallait, auparavant, plusieurs années pour dépouiller les résultats démographiques; une durée incompatible avec l'expansion d'un pays neuf en plein développement. Avec cette machine, l'État pouvait prévoir les écoles, les hôpitaux, les logements, et donc assurer une gestion des équipements publics compatible avec l'évolution démographique. La Tabulating Machine Company, firme fondée par Hollerith en 1896, s'associera en 1911 à d'autres compagnies sous le nom de Computing Tabulating Recording Company, qui deviendra en 1924 l'International Business Machines Corporation (IBM).

Parallèlement à la présentation de l'exposition dans la salle temporaire, le thème de la mesure irrigue la totalité du musée des Arts et Métiers, à

\footnotetext{
18. Marie-Agnès Bernardis, Bernard Hagene (dir.), Mesures \& démesure, Paris, Cité des sciences et de l'industrie, 1995 ; Witold Kula, Les mesures et les hommes, Paris, Éditions de la Maison des sciences de l'homme, 1984.

19. L'exemplaire exposé a été acquis par le Conservatoire des arts et métiers à l'issue de l'Exposition universelle de 1889 à Paris où il fut présenté (musée des Arts et Métiers-Cnam, inv. 11747). Il est conforme au brevet déposé par H. Hollerith, "Improvements in the Methods of and Apparatus for Compiling Statistics, Complete Specification n 327”, 8 janvier 1889, 3 pl. Une plaque située sur l'objet indique : The Hollerith Electric tabulating System / patented / in the United States / $\mathrm{N}^{\circ}$ 395781. JAN'Y, 1889. $\mathrm{N}^{\circ}$ 395782. JAN’Y, 1889. / Nº 395783. JAN’Y, 1889. / No / England, France, Germany, Austria Hungary, Belgium, Italy and Canada. / H. Hollerith, Washington, D.C., U.S.A.
} 
travers un parcours signalétique dans les salles permanentes à la découverte des instruments, étalons ou machines : aiguille de Vicat dans le domaine de la construction, roulements à billes instrumentés dans celui de la mécanique, lampe étalon Carcel dans la salle de l'énergie, etc. Ce parcours complémentaire permet de mettre en valeur des objets méconnus dont le rôle dans notre vie quotidienne a souvent été décisif.

Cette exposition est l'occasion de découvrir les richesses du musée des Arts et Métiers, mais aussi celles d'autres musées français ; ces instruments, souvent peu connus, sont cependant appréciables, soit pour comprendre l'histoire de la mesure, soit par leur caractère patrimonial. Mais qu'on ne s'y méprenne pas, ces objets authentiques, remarquables ou banals sont tous porteurs d'un message actuel. Ils n'existent que par ce qu'ils peuvent nous apporter aujourd'hui afin que nous puissions mieux comprendre le monde dans lequel nous vivons et les enjeux de la mesure dans la société du XXI ${ }^{\mathrm{e}}$ siècle.

\section{L'auteur}

Bruno Jacomy est le commissaire de l'exposition. Conservateur en chef honoraire du patrimoine, il a assuré la direction scientifique du Musée des Confluences, à Lyon, de 2005 à 2016. Ingénieur arts et métiers et docteur en psychologie sociale, il a été conservateur à l'Écomusée du Creusot-Montceau et directeur adjoint du musée des Arts et Métiers à Paris, responsable scientifique de sa rénovation. Historien des techniques et des instruments scientifiques, il a réalisé une douzaine d'expositions temporaires et a publié Une histoire des techniques, Paris, Seuil, 1990, nouv. éd. en 2015, et L'âge du plip : chroniques de l'innovation technique, Paris, Seuil, 2002.Contact : bruno.jacomy@free.fr 